Meta

Journal des traducteurs

Translators' Journal

\title{
Traduction et remotivation onomastique
}

\section{Barbara Folkart}

Volume 31, numéro 3, septembre 1986

Prismes de traductions littéraires

Facets of Literary Translation

URI : https://id.erudit.org/iderudit/002752ar

DOI : https://doi.org/10.7202/002752ar

Aller au sommaire du numéro

Éditeur(s)

Les Presses de l'Université de Montréal

ISSN

0026-0452 (imprimé)

1492-1421 (numérique)

Découvrir la revue

Citer cet article

Folkart, B. (1986). Traduction et remotivation onomastique. Meta, 31(3),

233-252. https://doi.org/10.7202/002752ar d'utilisation que vous pouvez consulter en ligne.

https://apropos.erudit.org/fr/usagers/politique-dutilisation/ 


\section{TRADUCTION ET REMOTIVATION ONOMASTIQUE}

BARBARA FOLKART

UNIVERSITÉ D'OTTAWA, CANADA

\section{ONOMASTIQUE ET SIGNIFICATION}

Sounds are impostures, déclare le Stephen Dedalus d'Ulysses. Like names, Cicero, Podmore, Napoleon, $\mathrm{Mr}$ Goodbody, Jesus, Mr Doyle. Shakespeares were as common as Murphies. What's in a name ?' 1 Au niveau de son dénoté, du moins, l'affirmation de Stephen Dedalus rejoint la théorie classique du nom propre : désignateur vide (R. Martin) ou rigide (Th. Pavel), qui réfêre mais ne signifie pas, structure double, circularité à l'intérieur de laquelle le code renvoie à lui-même et au référent sans fournir d'informations sur le compte de celui-ci, le nom propre est un signe arbitraire entre tous, du fait de la résistance marquée qu'il oppose au sens, une marge qui, même à l'intérieur de ce dire qu'est le texte, échappe à l'emprise de la signifiance.

Or l'in-signifiance proclamée ici de manière si péremptoire est niée par les connotés mêmes de l'énoncé : le propos de Stephen, écartelé par une antinomie flagrante entre les différents plans du contenu, semble construit pour se démentir. Ici, comme à maints autres endroits de l'œuvre joycienne, la praxis vient prouver que le nom propre est loin d'être in-signifiant : l'énumération onomastique proférée comme preuve de l'insignifiance démontre, au contraire, à quel point le nom peut servir de support à des connotés, surtout quand il est engagé dans un réseau de rapports tant paradigmatiques que syntagmatiques . Les noms allégués par Stephen se redistribuent en deux listes oppositionnelles, l'une composée des "illustres", Cicero, Napoleon, Jesus, l'autre regroupant les "inconnus », Podmore, $\mathrm{Mr}$ Goodbody, $\mathrm{Mr}$ Doyle; l'agencement syntagmatique en une alternance stricte des temps forts et des temps faibles déclenche un fonctionnement contrastif au niveau des connotés dont chacun de ces noms, resémantisé par les réseaux dans lesquels il se trouve engagé, devient le support - d'où la cocasserie d'une juxtaposition comme Jesus Mr Doyle.

Ainsi s'opère un processus de " communisation » qui fera du nom propre, non plus une entité réfractaire au sens, simple désignateur d'un référent unique, mais le support d'un signifié défini aussi bien en intension qu'en extension, un signe qui définit un type en même temps qu'il désigne des occurrences, qui exerce, pour reprendre une terminologie empruntée par Genette ${ }^{2}$, les fonctions déictique et épidéictique à la fois. Du référent-occurrence, du degré-zéro du signifié, l'on passe au signifié type, pour aboutir, au terme de la communisation, aux antonomases Shakespeares et Murphies.

\section{DONNER UN SENS AUX NOMS DE LA TRIBU}

Il s'agit en somme, même dans ce simple agencement d'appellatifs courants, d'une entreprise de remotivation onomastique : la marge préexistant au vouloir-dire est captée au profit d'un dire. En ceci, l'onomastique de l'œuvre littéraire ne fait que rejoindre la pratique poétique en général, qui est constitution d'un idiolecte, investissement de signifiés profonds dans des signifiants qui leur soient adéquats. En un mot, le propre du texte comme de l'onomastique poétiques réside dans le foisonnement des sens, dans la tenta- 
tive incessante pour remotiver le langage, pour triompher de la " perversité » des mots et des noms de la tribu.

Or, à première vue du moins, il semble y avoir une antinomie profonde entre motivation et traduction. N'est-ce pas l'opération traduisante, précisément, qui fournit la démonstration canonique de l'arbitraire du signe : puisqu'un même référent est désigné, en français par le signifiant [vaš] et en anglais par le signifiant [KAw], il ne saurait y avoir de lien analogique entre le signifié bovin et les signifiants qui dans les deux langues le prennent en charge. Inversement, dans la mesure même où le discours poétique tend à une adéquation du signifiant au signifié, l'on ne saurait récupérer celui-ci après avoir commuté celui-là. Et effectivement, le texte qui manifeste une motivation poétique oppose à la traduction immédiate l'obstacle souvent insurmontable de son opacité : le signifiant idiolectal se constitue en tant que tel, et se démarque de ses synonymes diasystémiques, précisément en exhibant la matérialité soit de son signifiant soit de son signifié ${ }^{3}$.

Or, l'antinomie entre traduction et motivation discursive sera levée si l'on envisage l'opération traduisante comme la réactualisation dans le texte cible $d u$ lien même de motivation qui constitue l'originalité du texte source. Tel passage d'Ulysses qui crie tout haut la motivation du signe :

\section{RHYMES AND REASONS}

Mouth, south. Is the mouth south someway? Or the south a mouth?

Must be some. South, pout, out, shout, drouth. Rhymes: two men

dressed the same, looking the same, two by two.

la tua pace

che parlar ti piace

... mentreche il vento, come fa, si tace

He saw them three by three, approaching girls, in green, in rose, in russet, entwining, per l'aer perso in mauve, in purple, quella pacifica oriafiamma, in gold or oriflamme, di rimirar fé più ardenti. But I old men, penitent, leadenfooted, underdarkneath the night : mouth south : tomb womb.

(ML : 136-137)

trouve son compte, pour l'essentiel, en français :

\section{RIMES ET RAISONS}

Bouche, couche. En quoi la bouche est-elle une couche? Ou la couche une bouche? Après tout. Couche, souche, touche, mouche, babouche. Les rimes : deux hommes vêtus de même, d'aspect semblable, deux par deux la tua pace che parlar ti piace

... mentreche il vento, come fa, si tace.

filles en vert, en rose, feuille-morte, enlacées ; per l'aer perso en mauve, couleur de pourpre, quella pacifica oriafiamma de l'or des banières, di rimirar fè più ardenti. Mais moi, tous les vieux pénitents, en semelles de plomb, parlenoirsous la nuit : bouche couche : antre ventre.

(GMD I : 200-201)

Le transfert est rendu possible par le fait que le signe idiolectal, remotivé, est un système de connotation à l'intérieur duquel il y a adéquation de l'" essence " du connotateur au connoté. Dans cette perspective, l'opération traduisante devient le transfert d'un certain nombre de pertinences fonctionnelles conceptualisables comme des traits suffisamment abstraits pour se laisser réactualiser dans un autre système linguistico-culturel. 


\section{LA MOTIVATION EXTRINSÈQUE}

L'entreprise qui consiste à doter de la bidimensionnalité du véritable signe cette entité unidimensionnelle qu'est le nom propre peut revêtir diverses modalités, selon que la motivation qu'elle instaure est de type extrinsèque ou intrinsèque.

\subsection{La sémantique du nom propre}

La motivation extrinsèque vise l'adéquation du signe onomastique-signifiant ou signifié- à son référent. Dans cette perspective cognomologique, il doit y avoir solidarité entre le nom et celui qu'il désigne, une convenance profonde entre la signification surajoutée et la désignation, en un mot, éponymie ${ }^{4}$ : «Barthes est Barthes parce qu'il s'appelle Barthes 》 et, inversement, nomina rerum consequentia sunt. Avec la motivation extrinsèque, c'est la sémantique du nom propre qui est mise en relief : la démarche éponymique débouche sur une nomenclature, affirme la primauté de la nomination. En même temps, elle programme une syntaxe virtuelle du nom propre, l'instaurant comme matrice actantielle dont la projection sur la syntagmatique engendrera le texte.

3.1.1 La manifestation la plus voyante du travail de motivation extrinsèque est donnée par le nom "construit " dans une visée éponymique. Il s'agit, comme l'a bien vu Fr. Rigolot ${ }^{5}$, d'une démarche onomasiologique consistant à remonter du faisceau de traits actantiels constitutif du personnage (le signifié de remotivation) au signifiant qui les prendra en charge. Autrement dit, il s'agit de construire un signifiant dans lequel puisse s'investir par surcodage le signifié de remotivation.

À l'intérieur de ce système de connotation, le référent peut être pris en charge soit par l'expression soit par le contenu du connotateur. Dans le micro-univers des Bijoux de la Castafiore, l'une des aventures de Tintin, un signifiant comme Bianca Castafiore est indiciel d'" italianicité " et (par renvoi à "Casta Diva ") d'" opéra ", deux des traits les plus pertinents du référent, tandis que Jean-Loup de La Batellerie est aristocratique à souhait ; à l'intérieur du couplet Dupond/Dupont, la quasi-identité des signifiants, variantes allomorphes d'un signifiant ayant déjà pour connoté "monsieur Tout-le-monde ", est homologue de la quasi-identité des référents. Le traducteur qui décide de naturaliser de tels noms propres dans le texte cible devra refaire ce parcours " onomaturgique " : dégager les traits qui sont pertinents du point de vue du fonctionnement discursif (démarche sémasiologique), puis les réinvestir dans un signifiant de la langue d'arrivée (démarche onomasiologique) - bref, choisir comme invariant translationnel non pas la manifestation, mais le trait latent. Ainsi, la projection sur l'anglais (l'excellent Castafiore Emerald) transforme l'aristocratique Jean-Loup de La Batellerie en le " classy-sounding» Christopher Willoughby-Drupe; Dupond avec d et Dupont avec $t$ devient Thompson and Thomson, with a ' $p$ ' and without (même si les traducteurs ne réussissent pas à rendre le brillant raccourci les Dupondt), tandis que Bianca Castafiore, invariant, retient son italianicité évocatrice de diva capricieuse et volatile, car les connotations attachées à ce type de nom existent aussi bien dans le système culturel anglais.

Alternativement, à l'intérieur de ce système surcodé qu'est le nom remotivé, le référent peut être pris en charge par le contenu : l'appellatif-descripteur - tout comme le terme technique qui lui aussi est dicté par un souci d'adéquation entre déixis et épidéixis - se présente volontiers comme le résidu d'une périphrase descriptive. Un patronymique comme Profitendieu, un sobriquet comme Skin the goat renvoient à des périphrases dont le dénoté est censé correspondre à une propriété du référent. Le Commendatore Bacibaci Beninobenone d'Ulysses manifeste un fonctionnement interlingual complexe : au niveau de l'expression, le jeu des sonorités sécrète les connotés « italianicité » et " grotesque "(du moins pour le récepteur non italien), tandis que le dénoté de la périphrase 
correspondante décrit soit une propriété présumée soit une antipropriété du référent (qui est, après tout, obèse et âgé). La traduction de tels condensés périphrastiques - à supposer qu'ils ne soient conservés tels quels - n'est pas sans rappeler le transcodage du terme technique : le signifié du descripteur technique ne recouvre pas l'ensemble des traits du dénotatum, de sorte que la traduction du terme peut amener en surface des pertinences négligées par le terme d'origine. De même, l'éponyme, le nom descripteur choisi dans la langue source, n'épuise pas forcément le faisceau actantiel qui définit le personnage : le traducteur est libre alors de substituer d'autres pertinences à celles retenues par le nom de départ si celles-ci ne se prêtent pas à une formulation suffisamment lapidaire dans la langue d'arrivée.

Les constructions onomastiques d'un Boris Vian constituent un cas limite, tant par la recherche qui a présidé à leur élaboration que par leur intégration à des textes qu'elles "programment ", pour ainsi dire. L'onomastique des romans, qui est invariablement récupérée par les structures signifiantes, apparaît comme " un système de production de fictions par traduction ou transcription ", souvent de mots anglais : l'Amadis de l'Automne à Pékin est un " Amadis des Buts ", tendu vers la réalisation de ses projets, programmé par une lecture en anglais du segment intertextuel avec lequel il polémise, "Amadis des Gaules"( $>$ goals) $)^{6}$.

3.1.2 Mais l'auteur peut choisir, plutôt que de construire une onomastique, de " donner un sens aux noms de la tribu» : le nom propre "ordinaire » peut devenir lui aussi le lieu où se manifeste une visée remotivante, pour peu qu'il soit récupéré par les structures signifiantes du texte qui l'accueille. Si Fr. Rigolot a pu parler dans ce cas d'" axe sémasiologique ", il s'agit, en fait, dans la mesure où le nom a été choisi par le sujet d'énonciation, d'une démarche onomasiologique : comme dans le discours technique, le signifié préexiste au signifiant et dicte le choix de celui-ci. Comme le remarque un Stephen Dedalus maintenant cratylien, à propos de l'onomastique shakespearienne : You will say those names were already in the chronicles from which he took the stuff of his plays. Why did he take them rather than others? (ML : 209). En somme, que les signifiants soient inventés ou simplement prélevés sur un fonds onomastique préexistant, l'encodage est une opération onomasiologique, même si le décodage par le lecteur - et par le traducteurlecteur - relève toujours de la sémasiologie.

Le plus souvent, c'est en tant qu'indice d'une appartenance sociale ou géographique que le nom propre ordinaire s'intègre aux structures signifiantes du texte, vocation qu'il est, en vérité, tout prêt à assumer : même en dehors de toute attache romanesque, le nom propre véhicule, comme connoté minimal, des informations sur le sexe, la nationalité, éventuellement le statut socio-professionnel, voire l'âge de son référent. Ce degré zéro du connoté se prête le plus naturellement du monde à des perfusions de sens. Le protagoniste principal d'Ulysses s'appelle Leopold Bloom : le judaïsme du nom est pertinent au plus haut degré, car il fonctionne comme connecteur entre ce juif errant dans le Dublin de 1904 et un prototype homérique supposé phénicien, donc sémite lui aussi. La toponymie dublinoise, exploitée dans le roman comme matériau phonétique et prosodique, devient en même temps un matériau signifiant dont l'utilisation massive porte à un paroxysme l'hibernicité d'un passage comme le suivant :

Before Nelson's pillar trams slowed, shunted, changed trolley, started for Blackrock, Kingstown and Dalkey, Clonskea, Rathgar, and Terenure, Palmerston park and upper Rathmines, Sandymount Green, Rathmines, Ringsend and Sandymount Tower, Harold's Cross. The hoarse Dublin United Tramway Company's timekeeper bawled them off :

- Rathgar and Terenure!

- Come on, Sandymount Green! 
Right and left parallel clanging ringing a doubledecker and a singledeck moved from their railheads, swerved to the down line, glided parallel.

- Start, Palmerston park!

(ML : 115)

Si le nom indice connote à travers son signifiant, le nom propre resémantisé de façon à mettre en valeur un signifié peut fonctionner comme un descripteur. Pareille resémantisation peut se faire par un cheminement étymologique qui reconduit le nom propre ordinaire au nom commun dont il est issu : les protagonistes du Bleeding Heart de Marilyn French s'appellent Dolores et Victor, elle parce qu'elle est une écorchée vive, lui parce qu'il sort vainqueur de toutes les confrontations où le place la vie. Dans la mesure où l'étymon est récupérable par la langue d'arrivée, ou même seulement par le système culturel d'arrivée (comme ce serait le cas de l'allemand, où douleur $>$ Schmerz mais où la connaissance d'un peu de latin suppléerait chez le lecteur à la perte du lien dans la langue d'arrivée), la remotivation étymologique conservera son rendement dans la traduction.

Citons, au moins pour mémoire, le cas où la sémantisation du nom propre ordinaire intervient au niveau de l'avant-texte et, de ce fait, ne laisse aucune trace à la surface du texte. Il en serait ainsi, dans $\dot{A}$ la recherche $d u$ temps perdu, du nom d'Odette, hypogramme in praesentia d'un paragramme in absentia : Odette = la cadette des cocottes connues. Pareil cas soulève des questions à tout le moins théoriques : l'opération traduisante peut-elle tenir compte de cette couche purement génétique ? Comment ?

\section{LA MOTIVATION INTRINSÈQUE : LA SYNTAXE DU NOM PROPRE}

La pertinence fonctionnelle de toutes ces onomastiques, leur rendement discursif se mesurent au degré d'interaction avec les autres structures signifiantes de l'œuvre. Audelà de la nomenclature, nous abordons la syntaxe du nom propre, c'est-à-dire la mise en forme de la substance onomastique, l'interaction du nom propre avec les autres signes, onomastiques ou non, qui sont engagés dans la signifiance du texte.

Nous avons vu comment, dans la boutade de Stephen Dedalus, même les « noms de la tribu " étaient remotivés par leur intégration à un système. Â plus forte raison, quand c'est au niveau des macrostructures romanesques que se tisse le réseau, entre noms au choix ou à la construction desquels a présidé un vouloir-dire, on assiste à un processus très marqué de motivation intrinsèque. De la nomenclature, à l'intérieur de laquelle le signe est aimanté par son référent, on passe au système signifiant, où le nom propre se trouve engagé grosso modo dans trois types de rapports syntaxiques.

4.1 Les rapports entre noms propres peuvent se manifester aussi bien sur l'axe paradigmatique que sur l'axe syntagmatique. Chez Proust, les noms entretiennent les uns avec les autres des rapports essentiellement paradigmatiques qui les partagent en deux séries de signifiants. On sait, en effet, que dans la Recherche, l'opposition " noblesse " vs " roture " s'investit dans deux systèmes formellement distincts, les sonorités alanguies signifiant " noblesse " tandis qu'un phonétisme " sec » signifie " roture». Le lien entre signifiant onomastique et signifié est conventionnel, mais une fois posée l'homologie :

$\frac{\text { Guermantes }}{\text { Verdurin }}=\frac{\text { des Laumes }}{\text { Bricot }}=\frac{\text { noblesse }}{\text { roture }}$


la sonorité du nom propre permet de le ranger dans l'un ou l'autre camp. Nous aborderons plus loin la projection sur la langue cible de ces séries signifiantes engagées dans un codage à motivation purement intrinsèque.

On retrouve par contre un fonctionnement aussi bien syntagmatique que paradigmatique dans Ulysses, qui fait un usage massif des virtualités signifiantes et ludiques du nom propre.

4.1.1 Le paradigme joycien s'organise autour d'un certain nombre d'oppositions basées surtout sur l'indicialité du nom propre. L'épisode du Cyclope, aimanté par l'atmosphère xénophobe qui règne dans la taverne-antre, met au premier plan des systèmes onomastiques polarisés par l'opposition hibernicité-non-hibernicité. Le terme non marqué est constitué par le tout-venant de la patronymique dublinoise : John Wyse Nolan, Paddy Dignam, Barney Kiernan, etc., pôle qui est axiologisé de façon positive par le nationalisme outrancier de certains personnages. Dans les contextes ultra-nationalistes intervient en plus une transformation destinée à exacerber l'hibernicité du nom par l'adjonction des particules $O^{\prime}$ ou $M^{\prime}$, qui marquent ainsi une sorte de degré superlatif : même l'insignifiant Alf Bergan se voit promu young chief of the O'Bergan's. Le terme marqué de ce système onomastique, victime, dans l'ensemble, d'une axiologisation négative, est constitué par le nom propre étranger, à la fabrication duquel Joyce s'en donne à cœur joie. L'étrangéité atteint son paroxysme dans le passage où est décrit, avec une faste onomastique qui engendre un grotesque puissant, l'exécution d'un jeune nationaliste irlandais ; on assiste à une prolifération hallucinatoire de noms extravagants en eux-mêmes (Commendatore Bacibaci Beninobenone, the Granjoker Vladinmire Pokethankertscheff, the Archjoker Leopold Rudolph von Schwanzenbad-Hodenthaler) ou rendus grotesques par leur fonctionnement syntagmatique. Comme la pertinence fonctionnelle de ce foisonnement onomastique réside tout entière dans son indicialité ethnique, il est évident en ce qui concerne la traduction que ces noms indices d'étrangéité (au même titre que les interjections polyglottes dont est émaillé le passage) doivent être maintenus tels quels dans le texte cible.

Un autre axe producteur de paradigmes onomastiques dans Ulysses est constitué par l'opposition "populo-gratin ", opposition qui se manifeste souvent, la xénophobie de certains personnages nonobstant, moyennant un surcodage qui fait du nom français le support du connoté "gratin". Ainsi, dans la réécriture in chiave mondana qui engendre, à partir du prototexte conifer family, tout un mariage sylvestre, le John Wyse Nolan de tous les jours resurgit, ennobli, sous les espèces du chevalier Jean Wyse de Neaulan :

The fashionable international world attended en masse this afternoon at the wedding of the chevalier Jean Wyse de Neaulan, grand high chief ranger of the Irish National Foresters, with Miss Fir Conifer of Pine Valley. Lady Sylvester Elmshade, Mrs Barbara Lovebirch ... graced the ceremony by their presence. ... On leaving the church of Saint Fiacre in Horto after the papal blessing the happy pair were subjected to a playful crossfire of hazelnuts. beechmast ... Mr and Mrs Wyse Conifer Neaulan will spend a quiet honeymoon in the Black Forest. (ML : 321-322)

Les ruminations de Bloom au sujet du beau monde trouvent leur point culminant dans l'évocation d'une Miss Dubedat dont la "gratinité ", à peine posée, est démolie par la juxtaposition avec Micky Hanlon :

Wouldn't mind being a waiter in a swell hotel. Tips, evening dress, halfnaked ladies. May I tempt you to a little more filleted lemon sole, miss Dubedat? Yes, do dedad. And she did bedad. Huguenot name, I expect, that. A miss Dubedat lived in Killiney I remember. Du, de la, French. Still it's the same fish, perhaps old Micky Hanlon of Moore street ripped the guts out of. (ML : 172-173) 
Tous ces noms deviennent ainsi le support de connotés qui fondent leur pertinence fonctionnelle à l'intérieur des macro- ou mésostructures du roman. Ces connotés doivent bien entendu être réactualisés dans le texte cible, et tout le problème de la traduction réside dans la recherche d'un support qui leur soit adéquat, qu'il reproduise ou au contraire remplace le matériel signifiant du texte source. Nous touchons ici à une question qui sera approfondie plus loin, celle de la naturalisation des onomastiques ou du maintien de leur altérité.

4.1.2 Tous ces paradigmes sont projetés sur l'axe syntagmatique. Le nom joycien, polymorphe, protéen, déploie des variantes hautes en couleur d'un bout du texte à l'autre : l'Irlandais tout-venant John Wyse Nolan s'épifie en O'Nolan, clad in shining armour, s'ajoutant un surcroît d'hibernicité avant de se métatextualiser, noblesse oblige, en le chevalier Jean Wyse de Neaulan, grand high chief ranger of the Irish Foresters. En sens inverse, that bloody poverty stricken Breen se mue en Signor Brini from Summerhill, the eyetallyano, papal zouave to the Holy Father. Le nom Leopold Bloom noyaute une constellation de variantes qui fluent et refluent, tout au long du roman, au rythme des assimilations/mimétisations/rejets d'un référent-protagoniste unique qui partage le sémitisme supposé et les errances de son homologue prototextuel, Ulysse. Bloom, nous l'apprenons, est une anglicisation - donc une mimétisation protectrice - du surnom paternel Virag (qui lui aussi veut dire "fleur", mais en hongrois). Dans la clandestinité de la correspondance "illicite" avec Martha Clifford, le mimétisme protecteur est poussé encore plus loin : Leopold Bloom devient Henry Flower. De mimétisation en mimétisation, notre héros passe de " juif + hongrois " à " juif + irlandais " à " gentil + irlandais ", allant jusqu'à figurer sous les espèces de O'Bloom, the son of Rory, bedight in sable armour dans une des interpolations de l'épisode du Cyclope. Naturalisation qui est démasquée périodiquement dans le roman : pour les litterati antisémites réunis à la bibliothèque au moment où Bloom y laisse sa carte de visite, la judaïcité mérite un appellatif encore plus congru : What's his name? Ikey Moses? dit un Buck Mulligan qui éprouve le besoin de vérifier la conformité du patronyme du visiteur avec sa physionomie. Dans le spasme d'antisémitisme qui clôt l'épisode du Cyclope, Bloom, chassé de la taverne, est apothéosisé en Ben Bloom Elijah, triomphe d'une judaïcité enfin revendiquée face aux insultes du Citoyen.

Dans l'ensemble, le traducteur français d'Ulysses a conservé telle quelle cette onomastique dont la symbolique est somme toute " internationale". À une exception près : l'archiassimilé Henry Flower devient Henry Fleury, comme pour parachever la mimétisation protectrice, en garantissant l'assimilation à un cotexte désormais français et par rapport auquel le nom n'a garde de se démarquer.

4.2 Les métatextualisations onomastiques d'Ulysses nous font entrevoir un deuxième type de relation syntaxique, les rapports du nom propre avec lui-même ou plutôt avec les interprétants qu'il contient ou engendre. Aussi bien les variantes paradigmatiques que les réécritures ludiques du "lemme" onomastique - anagramme, acrostiche, paragramme - ont en effet pour fonction d'expliciter la motivation du nom qui les noyaute.

4.2.1 Cette explicitation peut intervenir au niveau des interprétants amenés en surface par la variation ludique, le cas classique étant celui de la preuve par l'anagramme, l'acrostiche ou la paronomase. L'anagrammatisation, opérateur d'équivalence métalin-

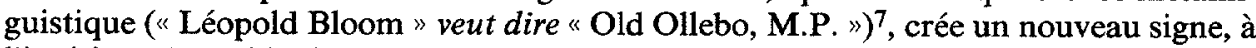
l'intérieur duquel le signifiant de départ est remotivé par le signifié qui surgit de son redéploiement : le nom propre, signe monolithique au départ, en rapport avec un référent 
mais dépourvu de signifié, devient le signifiant du contenu véhiculé par la réécriture anagrammatique. Comme l'a fait remarquer C. Kerbrat-Orecchioni ${ }^{8}$, le pouvoir de remotivation de l'anagramme tient à ce qu'il « resserre le lien sémantique qui existe entre les deux mots, qui sont en quelque sorte perçus comme deux avatars superficiels d'un même concept profond".

L'anagrammatisation pose des problèmes de taille au traducteur, parce qu'elle engage à fond la matérialité du signe. Quand, d'une part, le nom propre n'est pas engagé dans un réseau de signifiances et, d'autre part, le traducteur, optant pour une " naturalisation " du texte, a décidé de traduire l'onomastique, le cas est relativement facile et le traducteur peut se contenter d'une simple substitution de jeux de mots (ainsi la contrepetterie Turpin > Peinture, dans « Le retraité " de Boris Vian, rendue dans une traduction anglaise par le palindrome Nevols $>$ Sloven). Mais si le traducteur a décidé de garder l'onomastique d'origine (comme c'est le cas pour la majorité des noms propres dans la traduction française d'Ulysses), il est contraint ou bien de recourir à un jeu de mots interlingual ou bien de laisser tel quel l'anagramme initial, comme l'a fait Auguste Morel de crainte d'entraîner, de proche en proche, un bouleversement de tout le système onomastique bâti sur le nom de Leopold Bloom (Virag, Henry Flower, Poldy, etc.).

Un système paronomastique comme le couple formé par le terme de départ Leopold et son image Lionelleopold, engendrée par l'étymon leo, apporte une remotivation interlinguale qui survivrait dans un cotexte traduit dans une autre langue européenne. En revanche, une distorsion malveillante comme celle qui, proférée par un personnage qui trouve " chiante » la diva, transforme, dans la traduction anglaise, Castafiore en Castoroili, devrait, pour conserver son pouvoir de remotivation, être récréé de toutes pièces, au petit bonheur des moyens offerts par la langue d'arrivée (l'original français se contentait de la déformation non sémantisée Castafiole).

L'acrostiche constitue lui aussi une métatextualisation qui met en jeu la matérialité du signifiant. Comme l'a constaté Kerbrat-Orecchioni ${ }^{9}$, « seul le mot-thème (est) véritablement choisi et le reste du discours se contente d'en paraphraser les contours phonétiques et le contenu sémantique». Le traducteur en tirera les conséquences qui s'imposent.

Ainsi, dans Ulysses, le nom POLDY, diminutif de Leopold Bloom, engendre l'acrostiche suivant :

Poets oft have sung in rhyme

of music sweet their praise divine.

Let them hum it nine times nine.

Dearer far than song or wine,

You are mine, the world is mine.

Si la raison d'être du morceau, sa pertinence fonctionnelle résident dans le nom qu'il inscrit en vertical à l'initiale des vers, son sémantisme et sa thématique sont conditionnés de façon indéniable par ce nom en situation d'énonciation : alors que l'anagramme fonctionne comme une remotivation du nom qu'il transforme, l'acrostiche prend inévitablement l'allure d'un manifeste dont le signataire se sent solidaire corps et âme, son adhésion totale étant signifiée, de façon iconique, par l'imbrication des signifiants. Ainsi, dans le cadre d'énonciation fictif qui est celui de l'envoi par le jeune Bloom d'un billet doux à sa fiancée, le contenu engendré autour du nom POLDY se doit d'être celui d'une missive amoureuse ; dans le cadre d'énonciation au deuxième degré qu'est le roman dans lequel est enchâssée la missive, le contenu de celle-ci doit paraître teinté de sentimentalité, sa facture hypothéquée par des maladresses et des clichés, comme le veut la caractérisation du personnage. 
Il n'en reste pas moins que ce nom inducteur doit rester le grand invariant de traduction, que le texte en français doit se présenter avant tout comme une transformation du nom POLDY, ce qui demandera un réaménagement des dénotés à l'intérieur de l'enveloppe connotationnelle "sentimentalisme fade ", "versification d'amoureux de province" :

Puisque les bardes tous quand Phébus les inspire

Ont loué leur amour jusques au saint délire,

Laissons leur voix chanter jusqu'à ce qu'elle expire.

Depuis que tu es mienne, ô mon nectar, ma lyre,

Y a-t-il un empire égal à mon empire? (GMD. II : 389)

4.2.2 Au lieu de se manifester sur le plan du contenu grâce à des interprétantesgloses, la remotivation peut intervenir sur le plan de l'expression sous forme de variations ludiques qui sont homologues des variations subies par le support référentiel ; il s'agit alors d'une sorte de motivation extrinsèque dynamique. Dans tel passage hallucinatoire de l'épisode de Circe, les déformations de l'onomastique reproduisent, dans le matériau linguistique, les élongations et élargissements successifs d'un Bloom réfléchi dlans des glaces déformantes :

A concave mirror at the side presents to him lovelorn longlost lugubru Blooloohoom.... in the convex mirror grin unstruck the bonham eyes and fatchuck cheekchops of Jollypoldy the rixdix doldy. (ML : 426)

En fait, les déformations corporelles dans le sens de la longueur ou de la largeur sont doublées ici d'une dichotomie morale surgie comme une extrapolation du substrat prototextuel fat and jolly :

rotondité + jocosité $\longrightarrow$ maigreur + morosité.

- et il y a là un premier problème traductionnel, celui de la récupération d'un prototexte qui peut être sans homologue dans le système d'arrivée ${ }^{10}$.

Â partir de ces traits pertinents, l'adéquation du langage à ses référents se situe au niveau de la phono/graphostylistique, tant dans les segments onomastiques (maigreur graphique du $l$ tout en longueur, rotondité de l'o, mais aussi exploitation du phonostylème $l$, dont la liquidité renforce, par une référence possible aux larmes, les dénotés de longlost, lovelorn and lugubrious; allongement de la voyelle oo et récurrences du schème ol-p) que dans leurs prolongements par l'environnement sonore (fatchuck cheekchops, rixdix doldy). En même temps, il semble évident que le paragramme Blooloohoom passe par le relais de l'onomatopée boo-hoo-hoo (pleurs) pour s'adadapter à cette morosité supposée concomitante de la longueur, tandis que Jollypoldy passe par rolly-polly.

Si donc lugubru Blooloohoom et Jollypoldy recréent dans le matériau phonique et graphique des propriétés du référent, le puffing Poldy, blowing Bloohoom qui fait son apparition un peu plus loin (ML : 427) motive au niveau de la phonation, met en cuvre le matériau articulatoire de la langue, si l'on peut dire, dans la mesure où aussi bien les occlusives $p$ et $b$ que le fricatif $f$ exigent un mouvement phonatoire analogue au référent.

Toutes ces motivations exploitent la matérialité de la langue de départ, sa substance aussi bien graphique que phonétique et articulatoire. Leur transposition dans la langue d'arrivée devra passer par le détour d'une modélisation sémiotique, intuitive ou explicite, capable de mettre en évidence, par-delà le fonctionnement matériel, les pertinences les plus abstraites, celles qui ont le plus de chances de se réincarner dans un autre système linguistique.

Dans la traduction française il y a, inévitablement, perte de la strate prototextuelle : les syntagmes fat and jolly, rolly-polly, boo-hoo-hoo sont irrécupérables en tant 
que locutions figées en français. Le travail du traducteur devra alors porter en priorité sur la strate phono-graphostylistique. Or, le délaissé longtemps perdu lugubru Blooloohoom de la traduction française (GMD II : 132) relève davantage de l'équivalence sémantique (délaissé < lovelorn; longtemps perdu < longlost) que de l'équivalence phonostylistique (qui est présente, cependant, dans les isophonies en $l$ et en $u$ comme dans l'ululement évoqué par un Blooloohoom laissé inchangé). En revanche, le dans le miroir convexe s'hilarisent placides les yeux bamboches et les grasses joues et babouines d'ohéohépoldy le turlutututoldy fait une exploitation surabondante du matériau phonétique. Cependant, à y regarder de plus près, même un équivalent aussi réussi que ohéohépoldy le turlutututoldy est de nature sémantique plutôt que phonétique : le segment ohéohé provient, non pas des contours phonétiques du mot jolly, mais du connoté "chanson" ou " comptine » véhiculé aussi bien par la scansion du segment avoisinant, rixdixdoldy, que par le sémantisme de jolly, qui engendre le refrain par glissement métonymique (jolly chanson refrain ohéohê). La réussite de cette traduction tient davantage à son ludisme (et le ludisme est bien entendu l'un des traits les plus marquants du texte source) qu'à la motivation somme toute maigre qu'elle manifeste.

En ce qui concerne finalement les onomatopées phonatoires puffing Poldy et Blowing Bloohoom, l'on constate que le mouvement de phonation n'a été maintenu, dans la traduction française, que dans les items Poldy et poussif (Poldy poussif, Bloohoom qui fait des schproums, GMD II : 133); en dehors de son ludisme toujours à propos, le jeu sur Bloohoom et schproums paraît immotivé.

4.3 Un dernier type de relation syntaxique surgit entre le nom propre et les signes non onomastiques qui servent de support à la thématique du texte. Ce type de relation revêt une importance cruciale : tissé dans la trame même du texte, le nom propre acquiert un poids singulier, voit sa remotivation confirmée et intensifiée au point où, condensé de cette thématique, il devient le seul signifiant adéquat.

4.3.1 Il en est ainsi, dans The Bleeding Heart, du couple Dolores-Victor, que l'intégration à toute une série de mots thèmes installe dans une sorte de nécessité. Rendus à leur étymologie, les deux noms sont résorbés par les champs lexicaux actualisant les notions de " triomphe " et de " souffrance ", dont les hyponymes revêtent de ce fait le statut de mots témoins, d'indicateurs ou d'avertisseurs.

Or, cette intégration aux structures lexico-thématiques peut soulever des problèmes de traduction. Le nom Dolores est relayé, entre autres, par le syntagme bleeding heart, métaphore qui s'est lexicalisée dans la langue courante, où elle commute facilement avec des termes comme pinko, leftwing intellectual, starry-eyed libera ${ }^{11}$, mais qui, satellisée par le champ actantiel du roman et renforcé, comme dans les phrases suivantes, par des vocables comme draw the blood, bleeds, bruise, raw, est rendue à son sens propre :

A bleeding heart, that's what she was, although not the way he meant it.... But, God, what could you do? If you were one, you were one. Your only alternative was to avoid situations that would draw the blood. (p. 43-44)

"Now. What's wrong with your heart?"

"I told you. It bleeds. All the time. Goddamed bleeding heart... I'm like people who bruise if you even touch them, you know? ... Well, I have a soul like that, it's raw. » (p. 116-117)

Autrement dit, le syntagme bleeding heart fonctionne à la fois comme une simple désignation commutable avec d'autres et comme un allomorphe du nom Dolores. D'où l'inadéquation d'une traduction comme "les bons sentiments", qui ne relève que de 
l'isotopie "sensiblerie en matière de politique " et passe totalement à côté de l'isotopie générée par le nom Dolores (pain, weeps, priestess of pain), isotopie qui trouve son point culminant dans l'inqualifiable jeu de mots interlingual In French, "pain" means "bread" (p. 407).

Signe motivé doublement par les relations aussi bien syntaxiques que sémantiques qui se croisent en lui, désignation qui confine à la description en définissant un personnage dont il planifie et programme d'avance les interventions dans le récit, l'éponyme Dolores, ainsi que le Victor qu'il engendre, devient l'expression nécessaire et adéquate de l'actant qui le porte. C'est pourquoi on ne saurait lui substituer d'autres noms : à un certain niveau, The Bleeding Heart ne fonctionnerait tout simplement pas si ses protagonistes s'appelaient Mary et John. Le nom éponymique, comme le terme technique, n'est commutable avec nul autre : si le personnage de Dolores repousse avec tant d'agacement la transformation de son nom en Laurie par son amant, c'est parce que ce diminutif opérerait un changement de polarité (Laurie-laurels-lauriers du vainqueur-Victor) et consacrerait sa récupération par son opposé Victor.

4.3.2 La reconduction du couple Dolores-Victor à ses étymons débouche directement sur le champ lexical dont se tisse la thématique du roman. Mais l'articulation de l'onomastique sur la thématique peut être autrement complexe. Tel sous-ensemble de l'onomastique de la Nausée est motivé rétroactivement, de par son interaction avec la thématique du roman, à travers des structures sémiotiques fort élaborées, que nous avons tenté de dégager ailleurs ${ }^{12}$ et que l'on peut résumer comme suit :

\begin{tabular}{|c|c|c|c|c|c|}
\hline \multicolumn{5}{|c|}{ signifiants } & signifiés \\
\hline net & dur & immobile & passé & ... & + signification \\
\hline flou & mou & mouvant & présent & $\ldots$ & - signification \\
\hline
\end{tabular}

Un toponyme comme le boulevard Noir (qui présente, dans une occurrence sur-dix environ, la variante boulevard Victor Noir) n'est donné d'abord que comme simple désignateur. Cependant, le lieu qu'il marque, satellisé progressivement par une série lexicale on ne peut plus prégnante (dur, glacial, minéral, inhumain, pureté ...), s'investit vite du connoté " + signifiant » et, ce qui plus est, s'oppose à ces lieux du centre-ville où grouille une vie informe et qui se signalent au loin par la lueur rose dont les auréoles, réverbères et enseignes lumineuses : à travers des épaisseurs de noir, je distingue une pâleur rose : c'est l'avenue Galvani... Ici il n'y a que du noir'3'. (Entre ces lieux extrêmes, avec leur vide et leur trop-plein de vie, s'étend une zone de transition, la partie encore habitée du boulevard Noir, dont le trait distinctif est l'éclairage jaune de ses réverbères de plus en plus espacés.) Cette opposition de lieux est campée dans des termes d'une appartenance flagrante aux deux séries signifiantes dans lesquelles s'investit la thématique du roman :

\section{le centre-ville}

au cœur de la ville, aux grandes parures de feu des rues centrales

les choses vivantes, toutes les masses molles qui se meuvent spontanément

\section{le boulevard Noir}

J'ai dépassé le champ d'action du réverbère; j'entre dans le trou noir

Le trou souffle un vent glacial : là bas il n'a que des pierres et de la terre. Les 
les bruits domestiques, le ronflement des autos, les cris, les aboiements ne s'éloignent guère des rues éclairées, ils restent au chaud. pierres, c'est dur et ça ne bouge pas. Le boulevard Noir est inhumain. Comme un minéral.

J'ai froid./ Je suis gagné par la pureté de ce qui m'entoure; le vent siffle, des lignes raides fuient dans la nuit

La Nausée est restée là-bas dans la lumière jaune. Je suis heureux : ce froid est si pur, si pure cette nuit; ne suis-je pas moi-même une vague d'air glacé ?

Cette polarisation finit par sémiotiser l'opposition noir vs rose, qui est attirée à son tour dans la signifiance du roman :

$$
\frac{\text { noir }}{\text { rose }}=\frac{\text { absence de vie }}{\text { présence de vie }}=\frac{\text { simplicité }}{\begin{array}{c}
\text { foisonnement } \\
\text { et mouvement }
\end{array}}=\frac{+ \text { signification }}{- \text { signification }}
$$

Ainsi, par un détour à travers un champ lexico-thématique fortement perfusé, le très banal boulevard Noir devient-il l'un de ces "lieux dits justement " dont parle Ricardou : le nomen devient en vérité consequentia rei.

Si le traducteur préfêre, à une toponymie panachée ou transposée massivement dans la langue d'arrivée, une toponymie conservée telle que dans l'original, il devra faire confiance au bilinguisme au moins rudimentaire de son lecteur pour tirer au clair une opposition occultée par l'écartèlement de ses termes entre deux langues.

\section{LES SUPPORTS DE LA CONNOTATION À L'INTÉRIEUR DU SIGNE ONOMASTIQUE}

Les exemples que nous avons passés en revue dans la section précédente corroborent les critiques formulées par $\mathrm{C}$. Kerbrat-Orecchioni ${ }^{14}$ au modèle hjelmslévien de la connotation, qui, du moins dans la représentation devenue canonique, peut donner à croire que le rôle de connotateur est joué par le signe de dénotation en bloc :

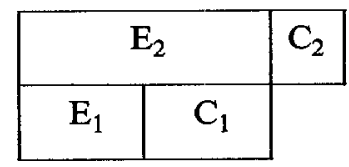

Des remarques de Kerbrat-Orecchioni, comme de nos exemples, il ressort que le support du connoté de remotivation peut très bien être une entité inférieure au signe, voire au signifié (il est tout à fait concevable que la récurrence de tel sème serve de support à une connotation littéraire) ou au signifiant (tout comme, dans le texte oral, une opposition dépourvue de pertinence linguistique - celle du $r$ roulé au $r$ grasseyé, par exemple - peut très bien être sémiotisée, de même, dans le texte écrit, la récurrence de traits inférieurs au signifiant minimal, voire au phonème, peut jouer le rôle que l'on sait dans l'homologation des plans de l'expression et du contenu).

Compte tenu de ces considérations, comme de la nature du lien qui unit le connoté à son support signifiant, nous résumons sous forme d'arborescence les modalités de connotation mises en évidence jusqu'ici pour le signe onomastique. Ne figurent dans ce 
schéma que des cas analysés dans cet article : il est clair que la branche signifiant, par exemple, pourrait être affinée de façon à inclure des unités signifiantes non autonomes, comme tel graphème $(\mathfrak{c}, \emptyset)$ typique d'une langue slave ou scandinave et, par là, indiciel d'une origine géographique).

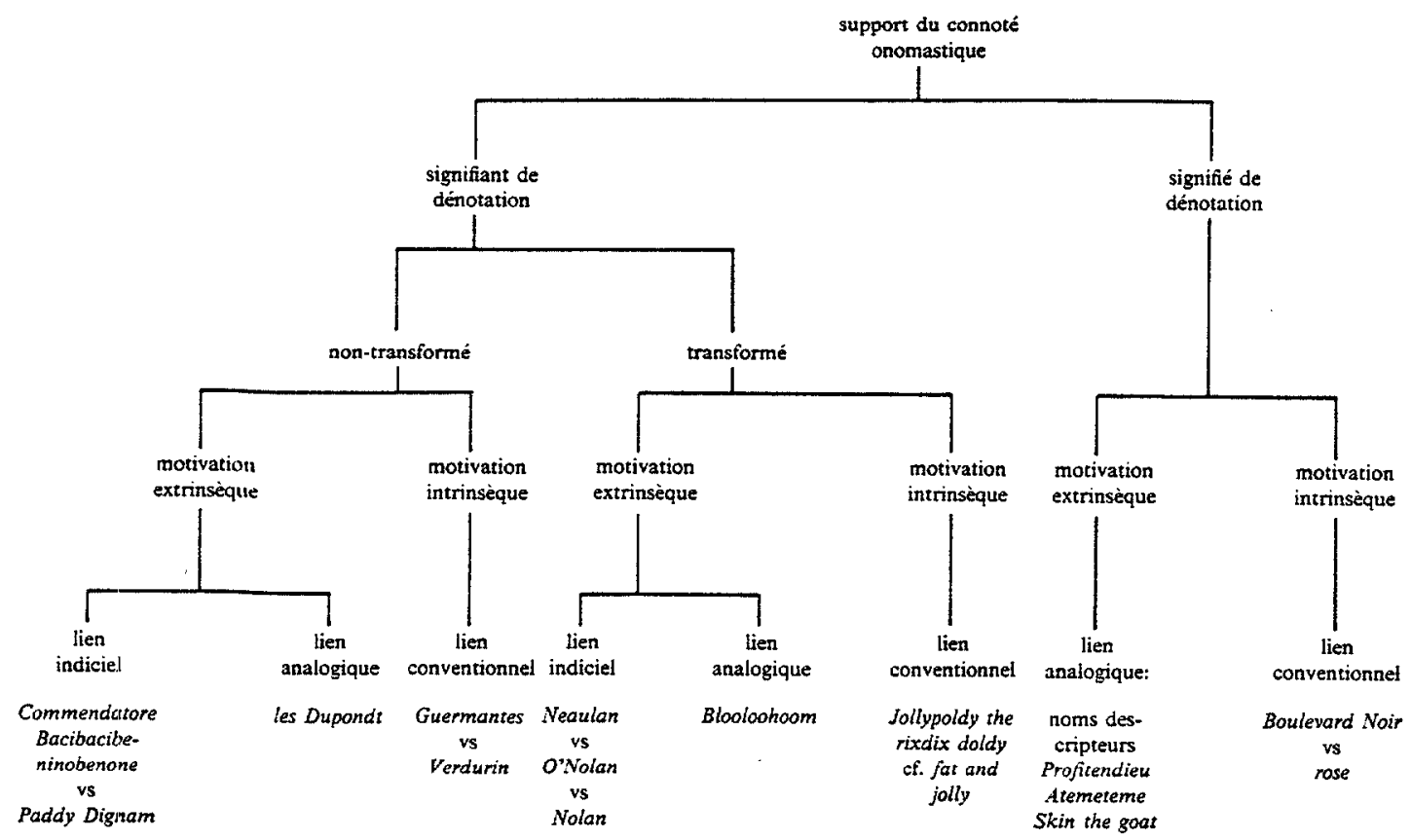

\section{CRITÈRES DE PERTINENCE ONOMASTIQUE}

Si l'onomastique peut être investie de façon à faire du nom un programme actantiel, thématique ou sonore, il n'en est pas toujours ainsi. Aussi importe-t-il de faire intervenir des critères pour décider de la pertinence, ou, au contraire de la non-pertinence de tel nom propre, étant donné que la projection translative est une réactualisation de structures fonctionnelles.

6.1 Un premier critère, plutôt quantitatif, est celui de la systématicité ou au contraire de la sporadicité des motivations investies dans l'onomastique. Le cas limite, comme toujours, est le texte de Boris Vian, où aucun nom n'est innocent et où la plupart doivent être appréhendés comme des matrices d'engendrement narratif. Sans être au même degré envahi par une motivation proliférante, un texte comme les Bijoux de la Castafiore - qui, rien que pour la qualité exemplaire de sa traduction anglaise, mérite de retenir notre attention - manifeste une systématicité assez prononcée, surtout au niveau des personnages de deuxième plan (les Dupondt, Tournesol) et des comparses qui, plus ou moins épisodiques (Jean Loup-de La Batellerie), ont besoin de "labels " permettant un repérage immédiat.

Il y a par contre des cas où la motivation onomastique - du moins celle qui se concrétise dans le texte, car nous n'avons aucune prise sur cette espèce de motivation existentielle qui fait que, pour un romancier comme Gide, il était impossible de dire grand chose d'un personnage qui n'avait pas encore reçu son nom définitif - il y a donc 
des textes dans lesquels la motivation onomastique semble être restée à l'état d'une velléité. L'onomastique des Faux-Monnayeurs, précisément, se scinde en une série de sousensembles qui pourraient bien fusionner en un système à fonctionnement contrastif mais qui en définitif restent en deçà de la systématicité. Le clivage le plus évident est celui qui oppose à l'onomastique du roman englobant, française dans l'ensemble, l'onomastique fantasque, gothique du roman en abyme. Mais des clivages plus subtils se dessinent à l'intérieur même du roman englobant, où des onomastiques indicielles d'" étrangéité " se démarquent par rapport à l'onomastique proprement française. Ainsi, au sous-ensemble étranger, qui regroupe avec des noms slaves (Boris, Bronja) ou anglais (Lady Griffith) des noms comme Ghéridanisol et Strouvilhou, dont l'étrangéité, moins localisable, devient le support d'une certaine "loucheté ", s'opposent des sousensembles protestant (Azaïs-Vedel) et "ultra-français ", l'archaïsme d'un nom comme Gontron de Passavant le portant au degré superlatif de la francité.

Mais ces démarquages morphologiques, quoique porteurs d'une certaine information, ne sont pas récupérés dans une visée de signifiance ; même la présence de certains noms à vocation descriptive (Profitendieu, Passavant, le couple Victor Laura) semble rester velléitaire. En somme, l'onomastique des Faux-Monnayeurs reste ambiguë. Les différents sous-ensembles, malgré leur différenciation morphologique et indicielle, ne constituent en fait qu'un "s-codice ", un signifiant en puissance que ne vient investir cependant aucun signifié qui serait pertinent du point de vue romanesque.

6.2 Manifestement, le critère d'une systématicité purement quantitative est insuffisant pour décider de la pertinence de telle onomastique romanesque. (Ce n'est pas non plus une condition nécessaire : le "système " du Bleeding Heart se réduit au seul couple Dolores-Victor, qui est néanmoins pertinent au plus haut degré.) Ce critère purement statistique doit être complété par un critère qualitatif et fonctionnel : celui de l'interaction de l'onomastique avec la thématique de l'ouvre. La pertinence du couple DoloresVictor est fondée par une interaction si prononcée que les noms propres sont résorbés dans le réseau de mots thèmes qui définit le texte. Le Mangemanche de l'Automne à Pé$k i n$ entretient avec le réseau textuel noyauté par la pseudo-auctoritas Lord Raglan citée en exergue les rapports labyrinthiques mis en évidence par J.-P. Vidal ${ }^{15}$. L'hibernicité ou la non-hibernicité du nom joycien est pertinente dans la mesure où cette opposition rejoint la thématique du roman (errances d'un Bloom-Ulysse sémite, xénophobie d'un Citoyen-Cyclope et, plus profondément, réflexion sur le mal spécifiquement irlandais dont est atteint ce Dublin de 1904). Par contre, la différenciation morphologique des sous-ensembles onomastiques des Faux-Monnayeurs, aussi poussée soit-elle, ne suffit pas à les intégrer à un système pertinent en l'absence de toute interaction avec les contenus profonds du texte.

En fait, les deux critères que nous venons d'examiner se complètent, ou plutôt le premier est subsumé par le deuxième. Il faut considérer comme un épiphénomène plus ou moins velléitaire tout nom qui, même s'il semble relever d'une motivation extrinsèque, reste sans écho à l'intérieur du texte, n'étant pas pris dans un système d'oppositions thématiques : ainsi, même s'ils se démarquent l'un de l'autre de par leur morphologie indicielle d'ethnicités diverses les différents sous-ensembles des Faux-Monnayeurs ne manifestent pas sur le plan du contenu un fonctionnement oppositionnel qui les rendrait pertinents du point de vue thématique et qui en ferait une substance intégrée à une macroforme. (Tout au plus suscitent-ils, autour du roman et en dehors de ses structures signifiantes, des relents désagréables de xénophobie et d'antisémitisme.) Est fonctionnellement non pertinent, en un mot, tout nom qui n'est pas engagé dans une syntaxe, que ce soit avec lui-même, les autres noms propres ou les signes constitutifs de la thématique. 
On retrouve là la primauté de la forme sur la substance. Tout nom qui ne manifeste pas une motivation intrinsèque, tout nom qui ne soit pas pris dans un réseau de relations syntaxiques à l'intérieur d'un système doit être considéré comme velléitaire même si en lui-même il est porteur d'un certain sémantisme. Profitendieu, Passavant, épisodiques, ne s'opposent à rien. Dolores est pertinent, non pas tant à cause de la motivation extrinsèque qu'il manifeste, mais parce qu'il s'oppose à Victor et, ainsi réétymologisé, rejoint toute une thématique ayant pour support le champ "bleeding heart".

\section{LA RÉACTUALISATION DES PERTINENCES ONOMASTIQUES}

Une fois reconnue la pertinence de telle onomastique surgit le problème de la réactualisation de ces pertinences dans la langue d'arrivée.

7.1 Une première dichotomie, qui ne se pose essentiellement que pour les onomastiques marginales (anthroponymes et surtout toponymes), fait intervenir la décision de garder ce connotateur qu'est le nom propre ou, au contraire, de le remplacer par un dénotateur non onomastique. H. Martinet ${ }^{16}$ signale, dans la traduction française d'un roman danois, le cas du toponyme Virum remplacé par le syntagme descriptif les bienpensants danois, mais il est évident qu'une troisième voie s'ouvre au traducteur, celle qui consisterait à remplacer Virum par le XVIe, Outremont ou Westmount, selon les cas, c'est-à-dire à naturaliser le nom propre, ce qui nous amène à notre deuxième point.

7.2 Une deuxième dichotomie, celle de la naturalisation ou, au contraire du maintien du nom propre dans son altérité, se pose pour le nom comme pour toute autre unité culturelle. Certes, on peut postuler que la norme en matière de transfert onomastique serait la traduction au degré zéro, consistant à conserver le nom dans son altérité, ne serait-ce que selon le principe d'un moindre effort producteur par surcroît de couleur locale, voire d'exotisme. Dans cette logique, il faut avoir des raisons pour traduire le nom propre, il faut être en mesure de justifier toute transposition de l'onomastique : si l'on ne peut pas, dans la langue d'arrivée, intégrer le nom substance à un système-forme, autant faire passer le nom tel quel dans le texte d'arrivée.

Mais même si ce principe s'avère sain dans l'ensemble, son application entraîne des pertes ou des ajouts dont le théoricien de la traduction se doit d'être conscient. Prenons un cas qui se particularise dès le départ de par son hybridisme linguistique, celui du texte littéraire africain écrit, comme il arrive le plus souvent, dans l'une des " grandes langues de culture " comme l'anglais ou le français. Une nouvelle comme "le Sermon de Yohannes Nkatafoé " de Guillaume Oyônô Mbia ${ }^{17}$ a la particularité d'enchâsser dans un texte narratif en français, comme un véritable texte dans le texte, un ensemble de noms africains construits comme des descripteurs : Atemeteme, c'est "celui-qui-estconstamment-étonné ", le père Mvendé-Nda-Zambe, c'est " celui-qui-maintient-l'ordredans-la-maison-de-Dieu ", etc. À la limite, une telle œuvre exige du récepteur une compétence bilingue/biculturelle boulou-française, faute de quoi le nom descripteur s'aplatit en un simple désignateur. Mais la problématique se complique du fait que le nom africain, dans son cotexte français, se charge de ce surcroît de sens qu'est son africanicité, voire, pour un lecteur non africain, son exotisme. Traduire ce texte pour un lecteur unilingue, anglophone, par exemple, revient à poser, à propos du nom propre, la question du sens à privilégier : puisque traduire, en pareil cas, c'est choisir, faut-il retenir la composante dénotative, narratologique, de cet Atemeteme qui veut dire "celuiqui-est-perpétuellement-étonné " ou la composante connotative, l'africanicité du nom? Selon qu'on aura opté pour l'africanicité ou pour la narrativité, l'on laissera le nom tel quel ou on le remplacera par un descripteur anglais. Dans le deuxième cas, l'on gommera cette solution de continuité entre nom propre et cotexte narratif qui était tout de 
même l'un des traits de l'original. Inversement, le traducteur qui maintient en français une toponymie comme celle de la Nausée finit par auréoler d'un certain exotisme un ensemble de désignations choisies justement pour leur banalité. Décidément, le texte cible, voué soit à abolir, soit à exalter l'altérité du nom propre, ne pourra jamais retrouver, par rapport à celui-ci, cette "juste distance " qui est celle du texte source.

7.2.1 Même quand le texte source ne se particularise pas par un trait aussi prégnant pour la traduction que l'hybridisme linguistique, la décision de traduire ou de ne pas traduire le nom propre, d'abolir ou au contraire d'afficher son altérité, doit toujours s'appuyer sur des critères fonctionnels. Certains cas se résolvent d'emblée. À un extrême, le nom dont la motivation passe par le signifié de dénotation, le nom descripteur pourvu d'une signification adéquate à sa désignation, doit être traduit, de façon à rendre ce signifié accessible dans le texte d'arrivée. À condition, bien entendu, que le signifié onomastique soit pertinent et non épisodique. Le cas limite de la pertinence onomastique est l'allégorie, où le nom, faisceau de signifiés dont la projection linéaire engendre le " récit ", doit être traduit. Les contenus onomastiques d'un Roman de la Rose traduit en anglais par Chaucer et, six siècles plus tard, par Ch. Dahlberg ${ }^{18}$, convergent à travers des signifiants marqués par l'évolution diachronique : Bel Samblant aboutit à FayreSamblant chez Chaucer et à Fair Seeming dans la traduction moderne, le Elde de Chaucer et le Old Age de Dahlberg remontent tous deux à Vielleice. Même en dehors de l'allégorie, le nom descripteur est porteur de contenus qui doivent être pris en charge par le texte d'arrivée : Skin-the-Goat, pertinent en tant que connecteur entre le métatexte Ulysses et le prototexte l'Odyssée (le personnage ainsi dénommé étant l'homologue du goatherd Eumée) est rendu en français par Peau-de-Bouc.

Dans les cuvres de Boris Vian, l'onomastique est partie prenante de la thématique et exige, plus qu'une traduction, une véritable transposition. Les aventures de Tintin, qui sont de partout et de nulle part, mettent en œuvre des systèmes onomastiques structurés bien plus par une typologie humaine et socio-professionnelle que par l'appartenance culturelle : eux aussi doivent faire l'objet d'une transposition suffisamment " libre » pour permettre à leurs signifiés de se réactualiser dans le système d'arrivée. Si la transposition de tout un système est licite, voire souhaitable dans les œuvres manifestant une forte intégration onomastico-thématique, la traduction d'un épiphénomène, par contre, comme le nom descripteur rendu épisodique par sa motivation purement externe, n'est nullement indiquée : la traduction de Profitendieu par Goodrich trancherait sur le reste du texte, entraînant, de proche en proche, un bouleversement injustifié de tout le microcosme familial et social dans lequel se déploie le roman.

À l'autre extrême, le nom qui est un pur indice d'origine ethnique, celui dont la motivation se manifeste donc au niveau du signifiant, doit être conservé tel quel, dans et pour son altérité : tel est le cas de l'onomastique hibernocentrique et de ses écarts " exotiques" (le chevalier Jean Wyse Neaulan, Commendatore Bacibaci Benininobenone) dans Ulysses, roman dont l'hibernicité est l'un des sens les plus profonds. Cette remarque présuppose, bien entendu - ce qui n'est évidemment le cas qu'entre cultures rapprochées - que les mêmes catégories d'ethnicité soient reconnues dans la culture d'arrivée et qu'elles s'actualisent à travers les mêmes marques, qui deviennent ainsi des indices à fonctionnement interculturel.

7.2.2 Si le nom propre qui fonctionne comme un indice d'appartenance ethnique doit être maintenu dans son altérité, il n'en va pas tout à fait de même du nom choisi ou construit pour signifier l'appartenance à une classe sociale. L'axe "noblesse" vs « roture », l'un des axes le plus fréquemment pris en charge par l'onomastique, peut être 
connoté, nous l'avons vu, par des signifiants soit indiciels, soit conventionnels. Dans le premier cas, c'est grâce à des marques qui varient nettement d'une langue à l'autre que le nom propre fonctionne comme indice de classe sociale : la morphologie connotatrice de "gratin" a un fonctionnement strictement intra-culturel. C'est ce qui légitime, dans le texte où le statut social du personnage est plus pertinent que son ethnicité, la transposition visant à substituer au signifiant d'origine un signifiant de nature à prendre en charge ces connotés dans la langue d'arrivée : Jean-Loup de La Batellerie devient Christopher Willoughby-Drupe. Certes, personne ne songerait aujourd'hui à toucher au système qui oppose Mathilde de La Mole à Julien Sorel, mais c'est là un phénomène qui, débordant le texte lui-même, intervient au niveau d'un cadre d'énonciation-réception à l'intérieur duquel les deux noms connotent, en plus de leur noblesse et leur roture respectives, "Stendhal » (c'est-à-dire "monument littéraire ") et "français ". La transposition onomastique a davantage de chances dans une cuvre qui, comme les Bijoux de la Castafiore, est moins distancée de par son prestige et de par son éloignement dans le temps.

Mais le prestige dont peut être auréolé le texte de départ n'est pas le seul obstacle à la naturalisation du nom propre indiciel de noblesse. Dans le couple qui oppose l'aristocratique Miss Dubedat au roturier Micky Hanlon, l'hibernicité l'emporte sur la classe sociale : autrement dit, Dubedat, tout en exploitant le surcodage du nom français, connotateur de "gratin ", reste un nom irlandais. Si, dans la traduction d'Auguste Morel, Miss Dubedat devient Mademoiselle de Saint Prix, c'est uniquement parce que le nom propre sert en même temps de support à une métatextualisation ludique :

Me ferez-yous le plaisir de reprendre de ces filets de sole-limande, Mademoiselle de SaintPrix? Je vous en prie. Et elle en prit sapristi. (GMD I : 253)

Le nom propre peut véhiculer des contenus comme "noblesse " ou " roture ", non pas en tant qu'indice, mais en tant que signifiant conventionnel, comme nous l'avons vu dans le cas de l'onomastique proustienne, partagée entre deux séries signifiantes caractérisées l'une par des sonorités alanguies, l'autre par des sonorités abruptes. Puisque le nom proustien est de toute façon construit comme un signe conventionnel et que, d'autre part, avec le recul du cadre d'énonciation opéré par la traduction, la francité de l'onomastique peut devenir un trait presque aussi important que le niveau diastratique, il n'y a vraiment pas de raison de remplacer les signifiants de départ par des signifiants tout aussi conventionnels mais prélevés sur le fonds onomastique de la culture d'arrivée.

\section{L'ONOMASTIQUE COMME LIEU DE DÉMARQUAGE}

Qu'il soit maintenu tel quel ou naturalisé, le nom propre constitue l'un des lieux où se révèle avec le plus d'acuité le démarquage entre texte source et texte cible. Conservé tel quel, le nom propre introduit une solution de continuité dans un cotexte auquel il est désormais hétérogène : Stephen Dedalus et Matilde de La Mole, homogènes avec leur cotexte d'origine, s'opacifient dans la mesure où ils tranchent sur une narration mise en français ou en anglais. Inversement, la traduction en anglais d'un roman comme le Ravissement de Lol V. Stein enlèverait à l'onomastique durasienne (Lol V. Stein, T. $B e a c h$, etc.) cette étrangeté mûrement préméditée qui la rend si prégnante dans l'original français : ici la distorsion provoquée par la traduction consiste à combler la distance voulue entre " texte» onomastique et cotexte narratif.

Les guillemets malencontreux qui entourent, dans la traduction française de The Women's Room de Marilyn French, des syntagmes comme Sever Hall ne font que propager au niveau de la typographie cette rupture plus profonde qu'est la frontière interlinguale : À présent, à l'âge de trente-huit ans, elle était blottie dans une toilette du sous- 
sol de "Sever Hall"19 (Toilettes pour femmes, p. 9). L'Irlandais Leopold Bloom compose, dans Ulysse, un acrostiche qui enracine tout un texte français dans le diminutif anglais Poldy, écrit sur son nom des anagrammes dont la remotivation est à ce point ancrée dans l'anglais que le traducteur a dû les laisser tels quels. La toponymie dublinoise, dont la musicalité et l'hibernicité étaient au plus haut degré pertinentes dans le texte source, devient, dans son nouveau cotexte français, un lieu de distanciation et, de musicale, devient exotique :

Devant la Colonne de Nelson les trams ralentissaient, bifurquaient, changeaient de prise, démarraient vers Blackrock, Kingstown et Dalkey, Clonskea, Rathgar et Terenure, Palmerston Parik et Upper Rathmines, Sandymount Green, Rathmines, Ringsend, Sandymount Tower et Harold's Cross. Le contrôleur enroué de la Compagnie des Tramways Réunis de Dublin braillait les départs.

- Rathgar et Terenure

- Sandymount Green, en voiture!

À droite et à gauche, parallèles, dans un fracas de sonneries et de coups de timbre, une voiture à impériale et une voiture simple quittaient leur terminus, inclinaient vers leurs voies descendantes, glissaient parallèles.

En route, Palmerston Park! (GMD I : 170)

En un mot, toute la distance entre nom propre et cotexte a été irrémédiablement et inéluctablement altérée.

Même traduite, l'onomastique creuse dans le texte cible l'une de ces failles à travers lesquelles se glisse ce surcroît de sens qu'est l'effet de traduction : mimétisé en gentil Irlandais, le juif irlandais Bloom signe Henry Fleury sa correspondance clandestine avec Martha Clifford; le Révérend Hugues C. Amour, licencié ès lettres, rencontré à Cahill's Corner, côtoie Patrick Dignam junior et Mme M.E. White, juste au coin d'Arran Street $W e s t$, dans le passage qui nous le montre en train d'adresser des salutations obséquieuses au compte de Dudley et à Lady Dudley (GMD I : 362-363). Lieu de contradiction, le nom propre traduit est une incongruïté qui frappe d'une invraisemblance aussi radicale que consentie tout le texte d'accueil, une incongruité que l'on ne saurait lever qu'en naturalisant, de proche en proche, tout le microcosme créé par le roman. En témoigne l'hybridisme foncier des personnages qui, dans la traduction anglaise de l'Étranger, mènent en anglais leur existence de petits-bourgeois pieds-noirs, hybridisme dont ne se débarrasserait le traducteur qu'au prix d'une refonte brutale qui supprimerait tout ce qui dans le monde du roman est en rapport d'altérité avec le nouveau cadre d'énonciationréception, et ce faisant atteindrait le roman dans ce qu'il a de plus essentiel : 1'invincibilité de son été méditerranéen.

À qui sait revenir sur des conventions de réception implicites qui escamotent l'altérité et tendent à installer le texte cible dans la même transparence onomastique que le texte source, cet hybridisme au niveau de l'onomastique, qu'il s'agisse de l'opacification du nom propre en rupture de système linguistique avec un nouveau cotexte ou de l'antinomie entre le nom propre naturalisé et les présupposés narratifs du texte, cette incongruïté inéluctable est en fait l'un des stigmates qui dénoncent dans la traduction le glissement du cadre d'énonciation. Distanciation par rapport à l'énonciation d'origine, la réénonciation opacifie ce qui dans l'énoncé d'origine était transparent, ouvre volontiers des perspectives métalinguistiques ou fausse la dimension métalinguistique de 1'origi- 
nal : la remarque de Bloom sur l'emploi de l'anglais dans la liturgie romaine renvoie, dans la traduction d'Auguste Morel, à un syntagme en ... français :

Enfin le prêtre s'agenouilla et commença de lire sur un carton :

- Ô Dieu notre refuge et notre force...

M. Bloom allongea le cou pour saisir les mots. De l'anglais. Leur jeter un os. (GMD I : 121)

\section{TRADUCTION, REMOTIVATION, RÉÉNONCIATION}

Le nom propre n'est que le lieu qui atteste avec le plus d'éclat, peut-être, le travail de remotivation dont est traversé le texte littéraire. Construit comme un système de connotation à l'intérieur duquel le signifié peut enfin s'investir dans un signifiant qui lui soit adéquat, le nom littéraire est lui aussi un signe idiolectal fabriqué pour porter remède à la perversité du langage, à ce défaut (à ce défi) qu'est pour le poète l'arbitraire du signe.

Et la remotivation du langage, tentative d'appropriation par le sujet parlant d'un système qui s'avère antisubjectif de par son caractère social même, constitue l'acte d'énonciation par excellence. Programme actantiel, thématique ou phonétique, le nom propre est l'un des lieux où s'inscrit dans le texte la subjectivité de l'énonciateur. En intégrant tels noms de la tribu, substance in-signifiante, à une forme qui leur confère un sens, l'énonciateur Stephen Dedalus marque d'un vouloir-dire son énoncé. Il a voulu que ce Doyle qui ne voulait rien dire dise quelque chose : de désignateur vide, Doyle, comme Podmore, Goodbody, Murphy, devient le connotateur du signifié "nullité ", tandis que les nébuleuses Cicero, Napoleon, Jesus, Shakespeare se condensent dans son énoncé, au contact l'une de l'autre, en un signifié plus pur : Cicero se réduit à la pure éloquence, Napoleon, Jesus, Shakespeare en viennent à ne plus signifier que " militarisme", " religion " et " génie artistique » (alors que toutes les nullités se valent en tant que degré zéro de la renommée). $A$ fortiori, quand le nom propre est construit ou choisi pour ensemencer tout un texte, il relève au plus haut degré d'une pragmatique.

Mais le nom propre constitue également le lieu où se manifeste avec le plus d'évidence l'altérité radicale du texte source et du texte cible : lieu de démarquage traversé de toutes les tensions entre deux textes et deux matrices culturelles, il atteste ce conflit d'énonciations qui est au cœur même de la traduction. La traduction est réénonciation, et ne peut que fausser, subtilement, le sens, qui, lui, est tributaire du cadre d'énonciation. Que le traducteur choisisse de conserver le nom et, avec lui, l'enracinement dans une culture donnée, ou au contraire d'en faire, dans une visée universalisante, un transfuge culturel, il ne pourra jamais le réénoncer tout à fait comme il a été à l'origine énoncé.

Notes

1. James Joyce, Ulysses (New York, Random House, 1934), p. 607. C'est cette édition, identifiée par le sigle ML, que nous citons tout au long de cet article. La traduction française est celle d'Auguste Morel (Paris, Gallimard, collection "folio ", 1983, vol. I et II); nos renvois à cette édition sont précédés des sigles GMD I ou GMD II.

2. Gérard Genette (1976) : Mimologiques, Paris, Seuil, p. 24.

3. Voir notre article, "Opacification and Semiosis : Ulysses as Double Structure ", à paraitre dans les Actes du troisième congrès de l'Association internationale de sémiotique.

4. Genette, op. cit., p. 24.

5. François Rigolot (1976) : "Rhétorique du nom poétique », dans Poétique 28, p. 469.

6. Nous nous sommes inspirée, pour tout ce qui a trait à l'onomastique de Vian, de l'étude consacrée par Jean-Pierre Vidal à "La déviance générale", dans le Colloque de Cérisy consacré à Boris Vian (Paris, Union générale d’éditions, 1977), vol. I, pp. 263-296.

7. François Goyet analyse le phénomène en termes grammaticaux : "Le nom propre est le thème dont l'anagramme est le prédicat ". ("La preuve par l'anagramme", dans Poétique 46 (avril 1981), p. 234.) 
8. Catherine Kerbrat-Orecchioni (1977) : la Connotation, Lyon, Presses universitaires de Lyon, p. 46.

9. Op. cit., p. 51.

10. Cf. notre article "Métatextualité et traduction", à paraître dans la Revue canadienne de littérature comparée.

11. Cf. "That's why I hate the liberal party line, it's such a godammed kneejerk response... - The bleeding hearts, you mean?" Marilyn French (1980) : The Bleeding Heart, New York, Ballantine Books, p. 76.

12. Voir notre article, "Translation as literary criticism", dans META 27:3, septembre 1982, pp. 241-256.

13. Jean-Paul Sartre (1960) : la Nausée, Paris, Gallimard, p. 41.

14. Op. cit., pp. 80-87.

15. Op. cit., p. 280.

16. H. Martinet (1982) : "Les noms propres dans la traduction littéraire", dans META 27:4, décembre, pp. 396-397.

17. Dans Chroniques de Mvoutessi I (Yaoundé, Éditions Cle, 1971), pp. 7-23.

18. The Romance of the Rose, traduction en prose de Charles Dahlberg (1971), Princeton, Princeton University Press.

19. Marilyn French, Toilettes pour femmes, traduction française de Philippe Guilhon (1978), Paris, Robert Laffont, p. 9. 